\title{
Atomistic simulation of fatigue crack growth in $\alpha$-Fe under high temperature
}

\author{
$\underline{\text { Tong Liu }}{ }^{1, *}$, Minshan Liu ${ }^{1}$ \\ ${ }^{1}$ Thermal energy engineering research center of Zhengzhou University, Zhengzhou 450002, China \\ * Corresponding author: liutung@zzu.edu.cn
}

\begin{abstract}
The crack growth behaviors loaded in mode I under strain and stress control at different temperatures were presented in $\alpha$-Fe by atomistic simulations using LAMMPS code. The interatomic bonds of atoms were characterized using the embedded atom method interatomic potential. The simulation models were built with initial edge crack subjecting to cyclic uniaxial constant strain rate and constant stress. A temperature range from $100 \mathrm{~K}$ to $1200 \mathrm{~K}$ was considered to probe the influence of the temperature on crack growth. The crack growth mechanism and the radial distrinution function (RDF) during crack growth were investigated. The results indicated that the crack propagation mechanisms were sensitive to temperature and the boundary conditions. By proposed image adjusting technology the dislocation slip bands can be more clearly displayed on screen. In order to include the effect of temperature on crack growth, a temperature factor defined as a function of temperature in exponential form was introduced to modify the theoretical expressions based on thermal activation theory. Its coefficent and index can be determined by the RDF peak value obtained from atomistic simulations. For cyclic loading the crack growth process was dependent on both temperature and cyclic loading period in terms of simulations.
\end{abstract}

Keywords Molecular simulation; Fatigue crack growth; High temperature; Iron; Thermal activation.

\section{Introduction}

Ferritic steels are widely used in process industry, modern nuclear power plants, military industry and others. The main crystal structure of steels is body-centered cubic (bcc) lattice of iron and it is comonly called $\alpha-\mathrm{Fe}[1]$. It is unavoidable for steels with defects so safety assessment on strucure integrity with defects is essential for engineering application. While in critical components of nuclear facilities, the radiation, high temperature and presssure, start and stop operations influence behavior of steels significantly and imply high potential failure risks for the integrity of reactor components with crack-like defects. The detail understanding of the processes occurring near the crack tip during the crack nucleation and growth can largely help in the material development and safety assessment under service in particular high temperature environments[2].

As the fatigue fracture is the primary cause of failure for almost all engineering structures subjecting to repeated cyclic loading, so many people have been do much research efforts on this scope. Most investigations on fatigue fracture have been performed at the macroscale and the theory on analysis methods and experimental approaches related to fatigue strength and fracture mechanics has been well established based on mechanics of continua and test at the macroscale with the crack lengths ranging from a few decades of microns to centimeters. However, on the kernel mechanism of fatigue crack initiation and growth are maily determined by interatomic bond rupture,crystal plastic deformation and the associated material defects in the vicinity of the crack tip. Recent developed atomistic simulations provide a high efficient approach to investigate fatigue fracture behavior at the nanoscale. With the knowledge of nanoscale fatigue, more accurate macroscale predictive tools can be got, mechanism understanding of grain boundary effects, crystal orientation effects, and driving force versus material resistance effects can be clearly obtained.

The material deformation and fracture mechanism of a structure with cracks have been paid close attention in worldwide. The fracture response of a material under mechanical load and temperature is a multiscale problem. It is the result of dislocation slip, grain boundary motion, plastic deformation and interplanar cleavage. The literature indicates that the atomistics of the crack 
growth in metals is considerablt complicated than current model postulation. The fracture mode of steels appears as ideal cleavage under low temperatures condition. The currently most widespread model for brittle crack growth in metals proposed by Rice and Thomson is that the high stress concentrations at the crack tip causes bond breaking in the cleavage plane and promotes dislocation emission from the crack tip. A successfully emitted dislocation will slightly blunts the crack tip. At very low temperatures the nucleation and/or removal of dislocations near the crack tip is suppressed, while at higher temperatures this constraint is relieved as a result of the thermally activated nucleation of dislocations. All these phenomena initiate at the atomic scale and form macroscopic consequences. As continuous advances on microscopic physics science and high speed development of computer technology, the molecular dynamics method is widely applied to analyze crack propagation mechanisms[3-14]. Utilizing molecular dynamics method not only a great deal of valuable micro-behaviors can be got but also the macro-properties of a material can be reproduced[15]. The behavior of crack growth in metals depends strongly on the structure evolution of the crack tip, load type and temperature. But at present it is still a lack of research on the influence of temperature on crack growth. The aim of our research was focus on the effect of temperature on crack growth in $\alpha$-Fe by using numerical simulations. For this purpose large scale molecular dynamics was applied to study the mechanisms of a crack growth under different temperature. Molecular simulations can reveal some crack propagation behavior in atomistic scale and offer a better understanding about crack growth. It even enhances an accurate evaluation method on crack growth and fracture behaviors at extreme temperature.

In present paper a model with constant rate of straining load in tension mode I and free surfaces was considered. Symetrical strain proportional to an atomic position in some dimension is imposed to all atoms. Periodicity along the thickness dimension $z$ was applied. Here atomistic simulations were applied to model the process of crack growth in steels under different temperature. The embedded atom method (EAM) potentials belonging to many-body interatomic potentials[1], which provide an essentially better description of the inter-atomic interactions and free surfaces, were employed to get accuracy results. The simulation parameters such as box geometry, timestep, loading rate were determined after performing parameter sensitivity analysis. The computation is implemented by utilizing large-scale atomic/ molecular massively parallel simulator (LAMMPS)[16], Visual Molecular Dynamics (VMD) [17] and developped programs in Python.

The simulation results demonstrated that with a crack growing the phenomena involving crack tip passivation, crack opening distance increment, dislocation emission, dislocation slip, plasticity deformation, voids formation and voids coalescence have been appeared and can be observed. Slip bands formation at the crack tips and ligament region and their development with incremental strain and temperature were demonstrated during crack growth. The crack growing with same initial state behaves differently under different temperature. It means that the crack growth trace and fracture configuration are realted with temperature. The dynamic radial distribution function and corresponding number density integral for variable time and temperature were calculated to explorer the relationship between the crack growth and temperature.

In this paper, the profile evolution of the crack tip for crack propagation system $\left(\begin{array}{lll}0 & 0 & 1\end{array}\right)\left[\begin{array}{ll}010 & 0\end{array}\right]$ in $\alpha-\mathrm{Fe}$ at low and high temperatures was investigated. It is expected to probe whether crack growth properties have relationship with some parameters referring to radial distribution function in $\alpha$-Fe during crack propagation at different temperatures. Some phenomenological descriptions on temperature-dependent relations allowing to predicting crack growth were proposed. The crack growth process, radial distribution function, atomic stresses in a bcc iron single crystal were analyzed utilizing molecular simulations with LAMMPS. Edge crack specimens under tentional strain loading were selected as simulation models. Limited by volume of the paper, here we only provide some key results. Other contents will be reported elsewhere.

\section{Crack growth and temperatrue}




\subsection{The effect of crystal plasticity on crack growth}

It has been known that the strength of metallic materials of various structures at extreme temperatures has a strong relationship with crystal plasticity generated by thermally activated microscopic mechanisms, which is usually controlled by elementary dislocation mechanisms. In order to understand and predict the crack growth and fracture behaviors of materials, it is extremely important to know what determine dislocation mobility and how it changes under the influence of stress and temperature. Based on thermal activation theory the derivation expression of the strain rate can be written by[18]

$$
\not E=\& \exp (-\Delta G / k T) \quad \text { with } \& \&=A \rho_{m} b\left(A^{\prime} / l\right) v_{i d},
$$

where $A$ is a geometrical coefficient, $\rho_{\mathrm{m}}$ is the mobile dislocation density, $b$ is the Burger' s vector, $A$ ' is the area swept by segment $l$ between two successive obstacles, $l$ is the wavelength of the vibration scales depending on the conditions, $v_{\text {id }}$ is the vibration frequency of the dislocation segment, $k$ is the Boltzmann constant and $T$ the absolute temperature. Here $\Delta G$ is the change of Gibbs free energy of the sample as the dislocation moves along dislocation coordinate. $\Delta G$ is used to characterize the energy barrier opposing dislocation motion.

In terms of fracture mechanics the crack growth is related with strain rate. Considering the above and from the point of view in engineering it prompts us to introduce a factor $C_{m}(\mathrm{~T})$ referring to thermally activated energy to take the effect of temperature on crack growth into account. Factor $C_{m}(\mathrm{~T})$ is defined as a function in form of

$$
C_{m}(T) \propto e^{-m / k T}
$$

where $\mathrm{m}$ is a constant which can be determined by molecular simulation.

\subsection{Radial distribution function (RDF)}

In statistical mechanics, the radial distribution function or pair correlation function $g(r)$ in a system of particles describes how the density varies as a function of the distance from a reference particle. It can also be determined experimentally, by radiation scattering techniques or by direct visualization for large enough particles via traditional or confocal microscopy. The radial distribution function is of fundamental importance in thermodynamics because the macroscopic thermodynamic quantities can usually be determined from $g(r)$. It is a primary reference for theory verification by experiments and also a fundamental function characterizing non-sequential system. For an ideal crystal structure the value and location of peaks at different near neighbour position indicates effect of temperature. Therfore it gives us an idea about whether it has realtionship with crack growth by means of thermal activation energy in different temperature. Based on the simulation results the atomic radial distribution function $\mathrm{g}(r)$ and the corresponding number integral are calculated and over a given trajectory.

\section{Molecular simulaton modeling}

\subsection{Interatomic potential}

An appropriate description of potential functions is essential in a molecular dynamics simulation. It concerns the accuracy and reliability of simulation results. Lots of potentials have been proposed to approximate the interactions of atoms, such as Lennard - Jones, Tersoff, embedded atom method (EAM) and etc. The steels studied for the crack growth simulation belong to amorphous metal model and is assumed to be made from Fe. The EAM potential for describing interatomic interaction in a semi-empirical form is used to study the crack growth of steels. It is suitable for characterizing metals and alloys with FCC, BCC and $\mathrm{HCP}$ structures. In a simulation, the EAM 
potential energy of an atom $\mathrm{i}$ is expressed by

$$
u\left(\left\{\mathbf{r}_{i}\right\}\right)=\sum_{i<j} \phi\left(\left|\mathbf{r}_{i}-\mathbf{r}_{j}\right|\right)+\sum_{i} F\left(\rho_{i}\right), \quad \rho_{i}=\sum_{j \neq i} f\left(r_{i j}\right)
$$

Where $\left|r_{\mathrm{i}}-r_{\mathrm{j}}\right|$ is the distance between atoms $i$ and $j, \Phi$ is a pair-wise potential function, $f(r)$ is the contribution to the electron charge density $\rho$ i from atom of $j$ at the location of atom $i$, and $F\left(\rho_{\mathrm{i}}\right)$ is an embedding function that represents the energy required to place atom $i$ into the electron cloud. Since the electron cloud density is a summation over many atoms, usually limited by a cutoff radius, the EAM potential is a multibody potential. The EAM of alpha iron used in present study comes from 'Interatomic Potentials Repository Project' which is included in potentials base of LAMMPS.

\subsection{Simulation models and methods}

The initial $\alpha-$ Fe planes were assumed to be perfect single crystal lattice having bec structures. The simulations were performed on major slip plane of bcc iron. Edge crack model was chosen to analyze the crack growth characteristics in an infinite domain under plane strain condition acquired by perform periodic boundary condition in $z$ dimension. The $x$-direction was defined forward in the crack plane, and the $z$-direction in the thickness direction. The schematic of the molecular dynamics simulation is shown in Figure 1. Three-dimensional model with a small thickness in order to reduce the calculation and to avoid the difficulty of the data analysis is constructed. The sizes of the simulation box were $858 \times 297 \times 5.7(\AA)$. It consisted of over 120000 atoms. There were two layers of transitional regions around the simulation domain. These layers were used to alleviate the effect of the boundaries and were not included in final results processing. The maximum positive and minimum negative strain loads were applied on the top and bottom boundaries at a certain strain rate to keep the crack faces apart (Figure 3). In all simulations, the initial crack was placed at the midpoint of the left side of the simulation lattice. And all related data including time step, coordinates, velocities, displacements, stresses, strains, temperatures etc. were saved.

The initial $\alpha-\mathrm{Fe}$ planes are perfect single crystal lattice having bcc structures was assumed in present paper. The simulations were performed on slip plane of bcc iron. The edge crack specimen geometry model adopted for the present simulation is illustrated in Figure 1. The initial crack was introduced by shutting off pairwise interactions between two slabs of atoms in perfect crystal to effectively create a sharp crack. The ratio of the initial crack length to the width of the specimen was $a_{0} / w=0.067$. The crystal orientation of the initial cracks in crack propagation system $(00$ 1)[010] was concerned and illustrated in Figure 2. Crack growth simulation was implemented by moving the atoms with the linearly varied velocity along y dimension in accordance with a given constant deformation rate, which is shown in Figure 3. The strain rate used in simulation was 0.005. A typical simulation cell with a pre-crack is drawn in Figure 4.

Surfaces on two transitional layers located at bottom and top constructed to alleviate the boundary effects were set free. The deformation loading was applied along $y$-direction perpendicular to the crack plane and varies linearly with $y$ coordinate. The constant strain rate loading was applied in such a way that the velocity is linearly distributed along the $y$-direction in core region. The velocity in transitional layer at the bottom and the top were set to the maximum positive and minimum negative value, respectively. The used loading method can effectively eliminate the stress oscillation and impact effect produced by acting sudden load. In the $z$-direction the periodic boundary condition was assigned to keep an expected plane strain state.

Another loading type used in simulation was to act forces directly on atoms in loading layers. External forces were applied in the way distributed in top and bottom surface boundary layers. The sizes of the simulation box are $578 \times 458 \times 2.86(\AA)$ which contains about 65000 atoms. Time step $0.002 \mathrm{ps}$ was utilized in simulations. The cyclic loading and unloading timesteps was 20000 . The initial crack length corresponds to 17.2,57.2 $\AA$. 


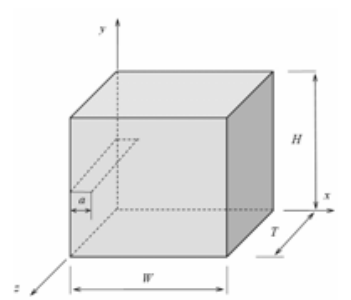

Figure 1. Schematic representation of the model to study the behavior of a crack growth

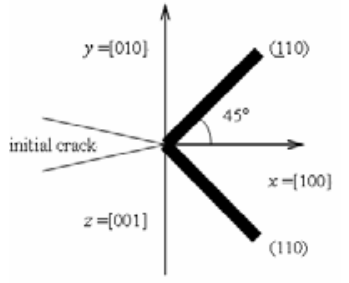

Figure 2. Crystallographic orientation of the studied initial crack

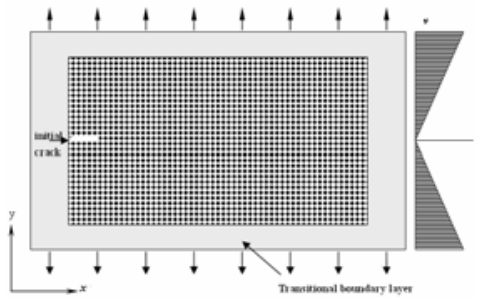

Figure 3. Edge crack specimen used for the simulation

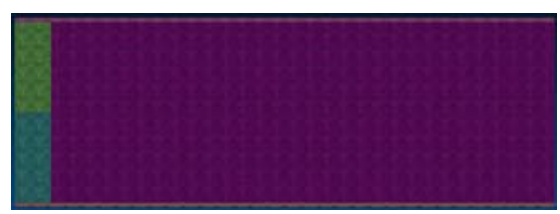

Figure 4. A typical simulation cell with a pre-crack

The temperatures in performed all simulations varies in the range of temperature keeping the iron in body-centered cubic crystal structure. The purpose of our research was to probe the influence of temperature on the crack growth behavior, thus the temperature of atoms in simulation was controlled to given value. The temperature of a group of atoms was reset by explicitly rescaling their velocities without performing time integration, unlike the NVT ensemble which performs Nose/Hoover thermostatting and time integration. It only modifies velocities that effect thermostatting. Therefor NVE ensemble using a separate time integration fix was utilized to actually update the positions of atoms using the modified velocities.

\section{Results and discussions}

\subsection{Direct observations of dislocation slip bands}

The dislocation as a result of atomic movement simualtion results can be shown in graphic mode through developped postprocessing code and VMD. In a general standard display style the dislocation and slip band stripes are not very clear and it is not easy to distinguish them. In terms of Moire interferometry method, an appproach to obtain clearer image of slip band patterns generated by deformed material crystals was put out in this paper by means of adjusting the drawing method, lattice node scale, plot size and screen grid resolution. This approach was called stripe image adjusting technique here. Figure 5 and 6 are two examples after applying proposed stripe image adjusting technique. By utilizing this new method the emission of slip bands from pre-crack tips can be observed clearly and picturesquely with the response of cracks propagating under congstant 
deformation rate control in the considered temperature range. However it needs more detail investigation to do quantitatively analysis because Moire patterns are sensitive to crystal lattice parameter, sphere scale and simulation cell sizes, as well as the screen grid intervals. The simulations demonstrate that slip bands started from the atomistically sharp crack tip. Simulations show that a slight blunting of pre-crack tips does not preclude slip band formation.

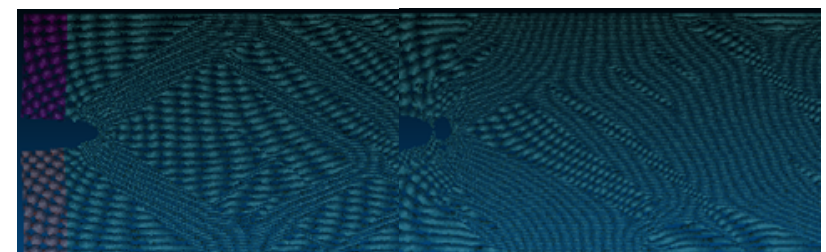

(a)

(b)

Figure 5. Dislocation slip bands for edge crack specimen at 300 K. (a) $t=3 n s$, (b) $t=5 n s$.

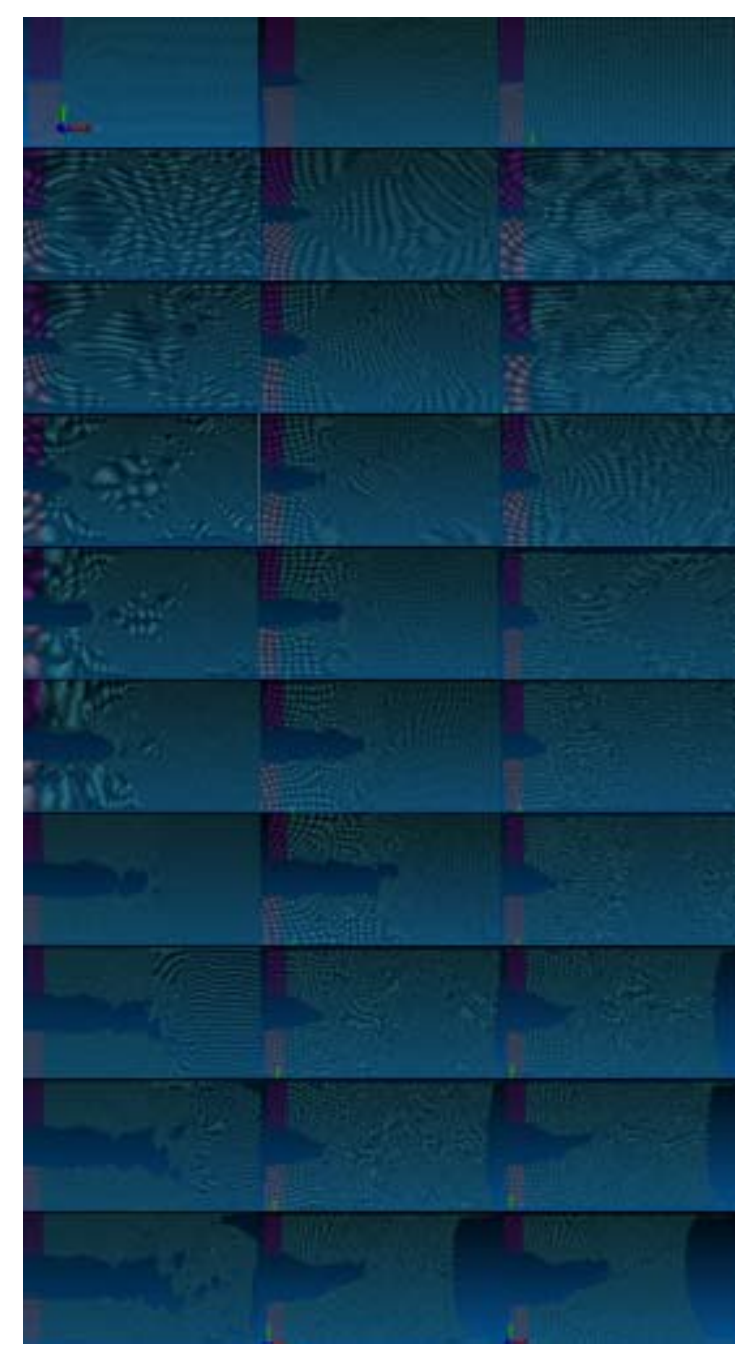

Figure 6. Intense dislocation slip bands at the crack tips.

A series of snapshots showing the crack growth at different time and temperatures were obtained in terms of performed molecular simulation results. The simulation time was measured in nanoseconds. Intense dislocation emssion and slip twin bands at the crack tip under different temperature $(\mathrm{T}=200$ $\mathrm{K}, 300 \mathrm{~K}, 600 \mathrm{~K}$ ) are illustrated in Figure 6. The slip bands evolving via time for present crack propagation system can be observed distinctly. The snapshots demonstrate the shapes and internal structure of slip bands near the tips of propagating crack. The slip band expansion paths were basically similar for simulation cases for given particular crack propagation system. The simulation 
results indicate that the crack growth modes under low and high temperature are different for their different brittleness and ductility. In a wide range of temperature the localized viscous flow, cavity formation and coalescence in ligament can be observed.

\subsection{Crack propagation processes}

Snapshots of propagating cracks under continuous strain loading at temperatures of $200 \mathrm{~K}, 300 \mathrm{~K}$ and $700 \mathrm{~K}$ are illustrated in Figure 7. All the phenomena involving dislocation, voids formation and merge were visually demonstrated. Figure 8 shows the crack growth difference for different temperatures at a given loading time. It is easy to see the crack propagating process and find that with same loading time duration the crack propagation rate reduces with an incremental temperature. The snapshots of dynamic crack growth also show that the ligament size and back boundary constraint condition influence the crack process and fracture profile considerably.

\subsection{Fatigue crack growth}

The cyclic loading was applied with a maximum stress $\sigma=50 \mathrm{MPa}$ and a stress ratio of $r=0$. External forces in the tensional direction were distributed in each atom located in top and bottom surface layers. The upper, lower and right boundaries were ensured constant displacement conditions in $y$ and $x$ direction respectively. The simulations were performed at a constant temperature of $300 \mathrm{~K}$ and $900 \mathrm{~K}$. In the case of initial crack length $a_{0}=17.2 \AA$ with $300 \mathrm{~K}$ and $900 \mathrm{~K}$ temperature cyclic simulations were performed and the crack propagation behavior is shown in Figure 9 and 10. The cracktip shape change and its moving process as well as dislocations can be observed. During the first ten cycles the crack grew slowly and then started to speed up. At sixteen cycles the crack penetrated the full section and specimen fracture occured. Based on the simulation results it is found that the crack growth rate were related to cyclic loading periods. Some more detail analysis should be done in future.

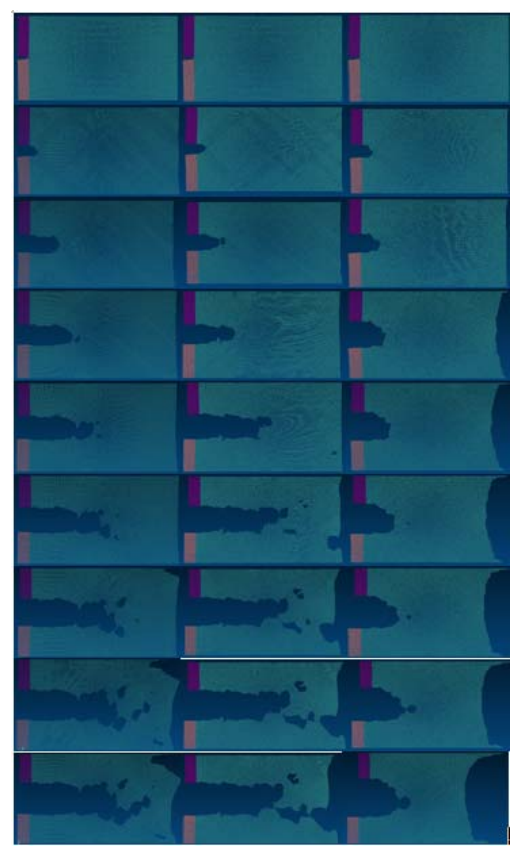

Figure 7. Crack propagation processes: Time $\mathrm{t}=1 \sim 15 \mathrm{~ns}$, Temperature $\mathrm{T}=200 \mathrm{~K}, 300 \mathrm{~K}, 700 \mathrm{~K}$

\subsection{RDF via crack growth}

Composing all the number density RDF integration curves under changing temperature and time 
cases and we obtained a plot as Figure 11. It is obvious that the variation or fluctuation is rather slight and implies that the simulation resutls have a good consistency. On the other hand it also supplys the used simulation model and parameters are appropriate. In addition, it illustrates that temperature has a weaker influence on RDF curve fluctuation than the crack growth process.

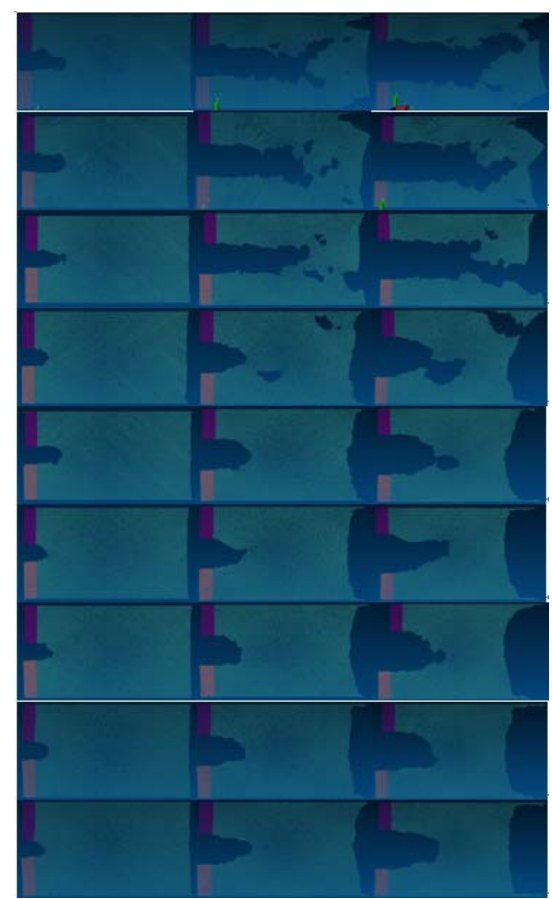

Figure 8. Crack propagation processes for Time $\mathrm{t}=5,10$ and $15 \mathrm{~ns}$,Temperature $\mathrm{T}=100 \sim 1200 \mathrm{~K}$

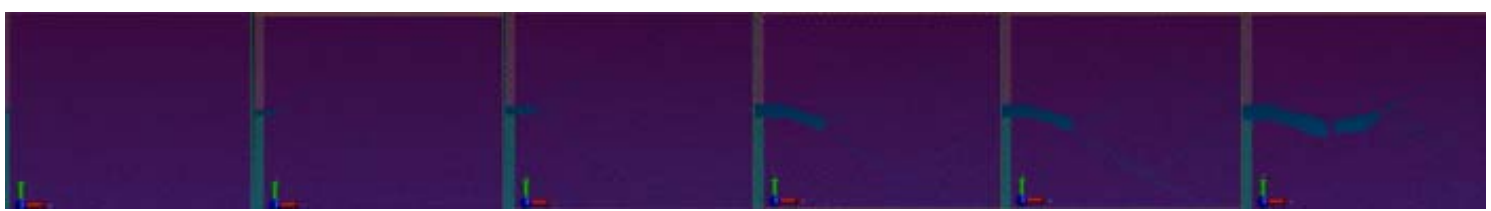

Figure 9. Crack growth process at $300 \mathrm{~K}$ with incremental load cycles $\mathrm{N}=10 \sim 16$

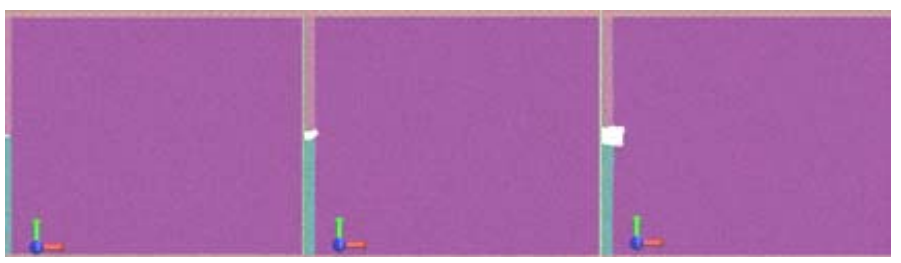

Figure 10. Crack growth process at $900 \mathrm{~K}$ with incremental load cycles $\mathrm{N}=10,15,20$

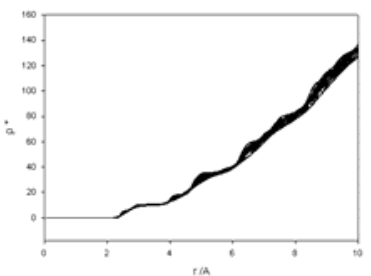

Figure 11. Number density integration curves

The RDF curves correspond to crack growth at $200 \mathrm{~K}, 300 \mathrm{~K}, 500 \mathrm{~K}, 700 \mathrm{~K}, 900 \mathrm{~K}$ and $1100 \mathrm{~K}$ are illustrated in Figure 12. It shows that the RDF curve reduces with increasing temperature. The changing patterns of maximum value or peak of RDF are plotted in Figure 13. All the curves have the same fixed oscillating modes but with different amplitude peak values. Only during the later stage of crack growth, there are a little phase shift. It is the results of large material plastic 
deformation.flow accompanying an increasing crack tip opening displacement. During fatigue cycles increase not only the crack size became largebut also the peak values of corresponding RDF curves reduced continuously.
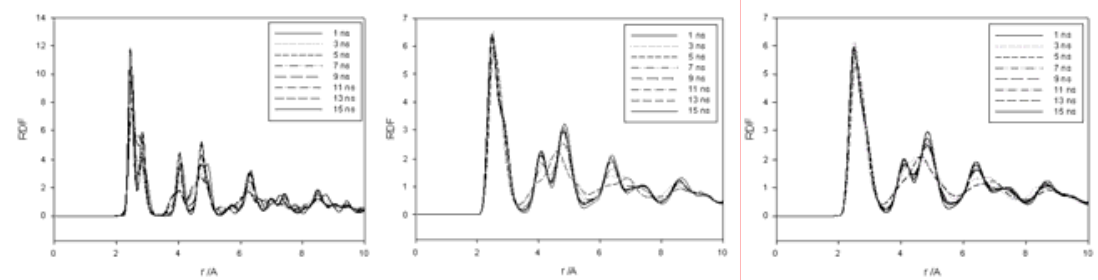

Figure 12. The RDF curves at $300 \mathrm{~K}, 900 \mathrm{~K}$ and $1100 \mathrm{~K}$

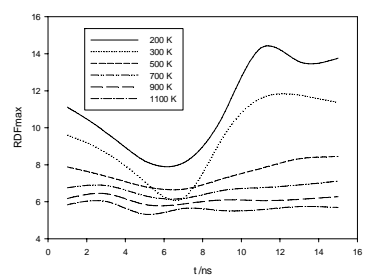

Figure 13. Relationship between the ultimate values of RDF and time

Figure 14 shows the RDF curves via temperature change at 5, 10 and $15 \mathrm{~ns}$ respectively. The ultimate values of RDF at different temperatures are plotted in Figure 15. Based on thermal activation theory and simulation results, the effect of temperature on crack growth can be taken into account by an expression in an exponential function form similar to expressions (1) and (2) which describes crystal plasticity. The coefficent and index values in expression are determined by the RDF peak value obtained from atomistic simulations. Here are some data processing results.

For time $5 \mathrm{ns:} C_{m}(T)=5.2191 e^{-\frac{99.53}{T}}$

For time $10 \mathrm{~ns}: C_{m}(T)=4.6203 e^{-\frac{234.89}{T}}$

, with correlation coefficient $\mathrm{R}=0.91$.

For time $15 \mathrm{~ns}: C_{m}(T)=4.9712 e^{-\frac{225.61}{T}}$

, with correlation coefficient $\mathrm{R}=0.99$.

with correlation coefficient $\mathrm{R}=0.97$

High regression correlation coefficients imply that the explanation and the used function form for considering the temperature influence on crack growth are appropriate.
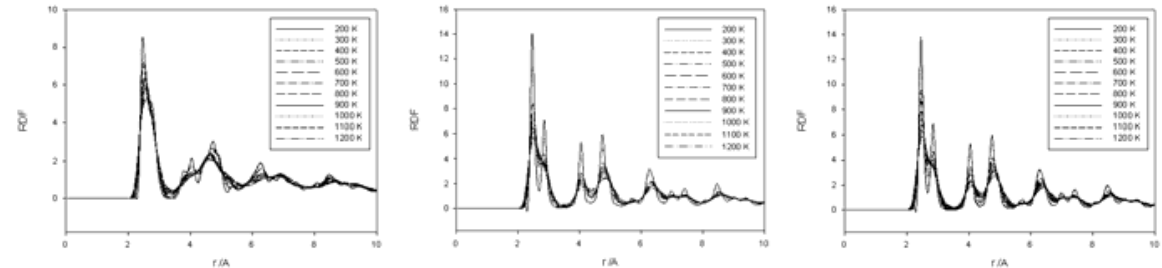

Figure 14. RDF curves via temperature change at $5 \mathrm{~ns}, 10 \mathrm{~ns}$ and $15 \mathrm{~ns}$

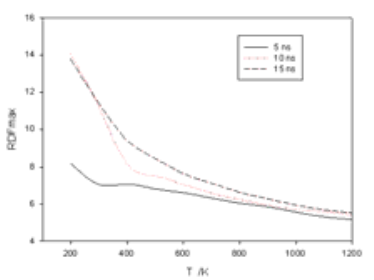

Figure 15. Relationship between the ultimate values of RDF and temperatures 


\section{Conclusive remarks}

In this paper crack growth behaviors and fracture in $\alpha$-Fe under different temperatures through molecular simulations have been investigated using Large-scale Atomic Molecular Massively Parallel Simulator code.

The mode I crack growth behaviors of an edge crack specimen under constant strain rate control at different temperatures have been investigated. A new image enhancement technique has been proposed to multiply the stripes of dislocation slip bands. It has been found that the temperature significantly affects the crack growth rate. As the temperature increases, the crack growth decreases. The results show that the mechanisms at the crack tip are sensitive to temperature and the boundary conditions.

The simulation results show that the crack growth behavior under cyclic loading was related to temperature, loading mode and cyclic loading period.

We performed atomistic simulations to study the relationship between RDF and crack growth behavior under a wide range of temperatures. Based on thermal activation theory and simulation results, the effect of temperature on crack growth can be taken into account by an expression in an exponential function form which describes crystal plasticity. The coefficent and index values in expression were determined by the RDF peak value obtained from atomistic simulations. The simulation results show that the crack growth process is highly dependent on the temperature.

\section{References}

[1] Wiki - Wikipedia, the free encyclopedia:en.wikipedia.org/wiki/Wiki

[2] J. L. Rempe and D. L. Knudson. High Temperature Thermal and Structural Material Properties for Metals used in LWR Vessels. Proceedings of ICAPP 2008. Anaheim, CA USA, June.2008.

[3] Ryosuke Matsumoto, Michihiko Nakagaki, Akihiro Nakatani and Hiroshi Kitagawa. MOLECULAR-DYNAMICS SIMULATION OF CRACK GROWTH WITH CRYSTAL NUCLEATION IN AMORPHOUS METAL. European Congress on Computational Methods in Applied Sciences and Engineering, ECCOMAS 2004.

[4] Yuan Gao, Cheng Lu, Guillaume Michal, Anh Kiet Tieu. A Study of Crack Propagation in BCC Iron by Molecular Dynamics Method. Key Engineering Materials, 385-387, 2008,453.

[5] Inga Ringdalen Vatne, etc..Quasicontinuum simulation of crack propagation in bcc-Fe. Materials Science and Engineering A 528 (2011) 5122-5134.

[6] V.A. Borodin, P.V. Vladimirov. Molecular dynamics simulations of quasi-brittle crack development in iron. Journal of Nuclear Materials 415 (2011) 320-328

[7] Ya-Fang Guo, Dong-Liang Zhao. Atomistic simulation of structure evolution at a crack tip in bcc-iron. Materials Science and Engineering A 448 (2007) 281-286.

[8] M.F. Horstemeyer, D. Farkas, S. Kim, T. Tang, G. Potirniche. Nanostructurally small cracks (NSC): A review on atomistic modeling of fatigue. International Journal of Fatigue 32 (2010) $1473-1502$

[9] Tian Tang, Sungho Kim, J.B. Jordon, M.F. Horstemeyer, Paul T. Wang. Atomistic simulations of fatigue crack growth and the associated fatigue crack tip stress evolution in magnesium single crystals. Computational Materials Science 50 (2011) 2977-2986.

[10] Tian Tang, Sungho Kim, M.F. Horstemeyer. Fatigue crack growth in magnesium single crystals under cyclic loading: Molecular dynamics simulation. Computational Materials Science 48 (2010) 426-439.

[11] D. Terentyev, E.E. Zhurkin, G. Bonny.Emission of full and partial dislocations from a crack in BCC and FCC metals:An atomistic study. Computational Materials Science 55 (2012) 313-321.

[12] Yue Fan, Akihiro Kushima, Sidney Yip, and Bilge Yildiz. Mechanism of Void Nucleation and Growth in bcc Fe: Atomistic Simulations at Experimental Time Scales. PRL 106, 125501 (2011) PHYSICAL REVIEW LETTERS. 
[13] Gao, Y, Lu, C, Tieu, AK Zhu. Molecular Dynamics Simulation of Crack Propagation on Different Slip Planes of BCC Iron. International Conference on Nanoscience and Nanotechnology, ICONN 2008,Melbourne, 25-29.

[14] Alena Uhnakova,Ann Machova,Petr Hora. 3D atomostic simulation of fatigue behavior of a ductile crack in bcc iron. International Journal of Fatigue. 33(2011) 1182-1188.

[15] Frenkel \& Smit. Understanding Molecular Simulation-From Algorithms to Applications. Academic Press. 1996.

[16] LAMMPS Molecular Dynamics Simulator: lammps.sandia.gov.

[17] VMD - Visual Molecular Dynamics, www.ks.uiuc.edu/Research/vmd/

[18] D. Caillard and J.L. Martin. Thermally Activated Mechanisms in Crystal Plasticity. London: PERGAMON, An Imprint of Elsevier,2003. 